\title{
Regionalgeschichte zum Mittelalter
}

\section{Ein Thema für Schulen in Schleswig-Holstein?}

Folgende Ausführungen stammen zwar nicht aus der Tastatur eines expliziten Fachdidaktikers, aber immerhin eines Regionalhistorikers mit gewissen fachdidaktischen Affinitäten. Die internationale Tagung „Bedrohte Landesgeschichte an der Schule?“, die auf meine Mitinitiative hin Anfang Dezember 2016 in Kiel stattfand, ${ }^{1}$ und der daraus hervorgegangene Tagungsband „Landesgeschichte an der Schule. Stand und Perspektiven“ aus dem Jahr $2018^{2}$ legen von dieser Affinität ein nach meinem Empfinden durchaus beredtes Zeugnis ab. Es sei hier gleich zu Beginn ganz prominent auf diesen Tagungsband verwiesen, weil er zur Thematik des folgenden Beitrags eigentlich den aktuellen Diskussionsstand wiedergibt, weswegen ich mich bei meinen Beobachtungen in ganz zentraler Weise auf seine Beiträge stützen werde. ${ }^{3}$ Dies gilt gerade für den Bereich Schleswig-Holsteins, auf den ich mich, der Denomination meiner eigenen Kieler Professur entsprechend, schwerpunktmäßig beziehen möchte. ${ }^{4}$ Allein acht der an jenem Band neben den beiden Herausgebern aktiv beteiligten 14 Autorinnen und Autoren rekurrieren in ihren Aufsätzen auf schulische bzw. fachdidaktische Erfahrungen und Gegebenheiten im nördlichsten Bundesland. ${ }^{5}$ Wegen der genannten Denomination und der zeitlichen Ausrichtung der hiermit vorgelegten Veröffentlichung gemäß sei im Folgenden das Hauptgewicht auf mittelalterliche Regionalgeschichte Schleswig-Holsteins als schulisches Thema gelegt. Dies ist besonders deswegen hervorzuheben, weil Landes- und Regionalgeschichte im Schulunterricht, wenn überhaupt, eher im Rahmen der neueren und Zeitgeschichte zur Kenntnisvertiefung und -erweiterung der Schüler*innen auf der Grundlage

\footnotetext{
1 Siehe den Tagungsbericht: Anna Brauer/Marvin Groth/Markus Wilke, Bedrohte Landesgeschichte an der Schule? Stand und Perspektiven, 01.12.2016 - 02.12.2016 Kiel, in: H-Soz-Kult, 8.3.2017, www.hsozkult.de/conferencereport/id/tagungsberichte-7044 (letzter Zugriff: 10.3.2020).

2 Oliver Auge/Martin Göllnitz (Hrsg.), Landesgeschichte an der Schule. Stand und Perspektiven. Ostfildern 2018 (Landesgeschichte, Bd. 2).

3 Siehe daneben ebenfalls aktuell und grundlegend Anke John, Lokal- und Regionalgeschichte. Frankfurt a. M. 2018 (Methoden Historischen Lernens); vgl. außerdem noch - mit eher defensiver Argumentation - Dietmar Schiersner, Alter Zopf oder neue Chance? Regionalgeschichte in Historiographie und Geschichtsunterricht, in: GWU 62, 2011, 1/2, 50-60; schon älteren Datums, aber immer noch zitabel Karl-Hermann Beeck (Hrsg.), Landesgeschichte im Unterricht. Ratingen 1973 (Schriftenreihe zur Geschichte und politischen Bildung, Bd. 11).
}

4 Die Denomination lautet: Professur für Regionalgeschichte mit Schwerpunkt zur Geschichte Schleswig-Holsteins in Mittelalter und Früher Neuzeit.

5 John, Lokal- und Regionalgeschichte (wie Anm. 3), 65, lässt Schleswig-Holstein leider außen vor.

> Das Werk ist unter der Creative-Commons-Lizenz Namensnennung - Weitergabe unter gleichen Bedingungen 4.0 International veröffentlicht. Den Vertragstext finden Sie unter: https://creativecommons.org/licenses/by-sa/4.0/deed.de. Bitte beachten Sie, dass einzelne, entsprechend gekennzeichnete Teile des Werks von der genannten Lizenz ausgenommen sein bzw. anderen urheberrechtlichen Bedingungen unterliegen können. 
des Vergleichs genutzt wird. ${ }^{6}$ In einem ersten Abschnitt wird es dabei um die derzeitige Ausgangslage, in einem zweiten um die künftigen Potentiale gehen, die sich diesbezüglich für eine schulische Vermittlung von mittelalterlicher Regionalgeschichte bieten.

\section{Die Ausgangslage: Regionalgeschichte zum Mittelalter - ein Thema an schleswig-holsteinischen Schulen?}

Mögliche allgemeine Vorteile eines regionalhistorischen Unterrichts sind vielfach behandelt ${ }^{7}$ und seit langem benannt, wie ein Beispiel aus dem südwestdeutschen Landkreis Göppingen von 1979 zeigt: $^{8}$ In der dort publizierten Einleitung zu einer regionalhistorischen Quellen- und Textsammlung für den Geschichtsunterricht wird auf die besondere „Objektnähe“ des Regionalen, die die Geschichte konkreter und überschaubarer, die Schüler*innen betroffener und den Unterricht experimenteller mache, abgehoben. Das Spektrum des methodischen Vorgehens werde zudem erweitert, z. B. durch Exkursion und Erkundung, und obendrein werde eine höhere Lerneffizienz bei gleichzeitiger Reduktion lernpsychologischer Widerstände gegen das Fach Geschichte als solches erreicht. Regionalgeschichte, so heißt es weiter im Text, trage zur geistigen und emotionalen Verwurzelung der Schüler*innen bei und wirke in Zeiten zunehmender gesellschaftlicher Polarisierung integrierend. Regionalgeschichte motiviere die Schüler*innen zum selbständigen Erkunden der eigenen Umgebung. Und zuletzt: „Regionalgeschichte ist ein legitimer Zugang gerade zu einem Verständnis der deutschen Geschichte, die sich über lange Zeitstrecken nicht in übergeordneten Zentren machtpolitischer Entscheidungen, sondern in regionalen Bereichen der Herrschaften, Territorien und Städte abgespielt hat.“ Zweifel an der besonders motivierenden Funktion der Regionalgeschichte, die in der gerade erwähnten Einleitung unterstrichen wird, seien nicht verschwiegen. Umfragen unter Schüler*innen ergaben vielmehr ein niedrig

\footnotetext{
6 Siehe hierzu zusätzlich, allerdings am Beispiel Mecklenburg-Vorpommerns: Anke John, LG - Lokal-, Regional- und Landesgeschichte in den Lehrplänen Mecklenburg-Vorpommerns für den Geschichtsunterricht, in: Dies. (Hrsg.), Köpfe. Institutionen. Bereiche. Mecklenburgische Landes- und Regionalgeschichte seit dem 19. Jahrhundert. Lübeck 2016 (Veröffentlichungen der Historischen Kommission für Mecklenburg, Reihe B, N.F.: Schriften zur mecklenburgischen Geschichte, Bd. 5), 257-270, hier 265. Diese Feststellung kann cum grano salis aber auch auf den Unterricht in Schleswig-Holstein mit seiner Prädominanz der Beschäftigung mit Zeitgeschichte übertragen werden. John verweist in diesem Kontext noch auf Helmut Beilner, Heimatgeschichte als Regional- und Lokalgeschichte, in: Waltraud Schreiber (Hrsg.), Erste Begegnungen mit Geschichte. Grundlagen historischen Lernens. Bd. 2. Neuried 2004 (Bayerische Studien zur Geschichtsdidaktik, Bd. 1.2), 859-883, hier 874f. („exemplarische Funktion"). Vgl. des Weiteren Bernd Schönemann, Regionalgeschichtlich akzentuiertes historisches Lernen. Eine Bestandsaufnahme in systematisierender Absicht, in: BlldtLG 144, 2008, 1-11.

7 Peter Knoch, Überlegungen zu einer Didaktik der Regionalgeschichte, in: Ders./Thomas Leeb (Hrsg.), Heimat oder Region? Grundzüge einer Didaktik der Regionalgeschichte. Frankfurt a. M. 1984 (Geschichte lehren und lernen), 3-16, hier 14-16. - Rolf Schulte, Didaktik einer Landes- und Regionalgeschichte, in: Demokratische Geschichte 21, 2010, 197-208.

8 Vgl. dazu und zum Folgenden: geschichte regional. Quellen und Texte aus dem Kreis Göppingen, Heft 1. (Hohenstaufen, Folge 11) Göppingen 1979, 4 (Einleitung).
} 
ausgeprägtes Interesse an regionalgeschichtlichen Themen. ${ }^{9}$ Doch sind die Studien für sich genommen nicht unproblematisch, da ihnen ein Zirkelschluss inhärent zu sein scheint. Kann man bei Schüler*innen von vornherein ein stark ausgeprägtes Interesse an Regionalgeschichte erwarten, wenn im elaborierten Unterricht der Blick auf größere Raumeinheiten dominant ist? Oder umgekehrt gefragt: Waren die Resultate der Befragungen - eine Präferenz der National-, europäischen oder Globalgeschichte seitens der Schüler*innen - angesichts der Schwerpunktsetzungen im Unterricht nicht ohnehin vorhersagbar? Hier scheinen weitere empirische Untersuchungen nötig zu sein, um zu einer fundierten Klarheit in dieser Frage zu gelangen.

Noch vor wenigen Jahren oder doch besser Jahrzehnten war jedenfalls die schulische Welt für den schleswig-holsteinischen Landes- und Regionalhistoriker und sein weibliches Pendant, rein formal betrachtet, in bester Ordnung. In den seit der Mitte der 1980er Jahre geltenden Lehrplänen war die Beschäftigung mit landes- und regionalgeschichtlichen Themenfeldern im Schulunterricht mehr oder minder konkret vorgegeben. Und selbige machten den Lehrkräften ebenso konkrete Vorschläge für einen landes- oder regionalgeschichtlich ausgerichteten Unterricht. ${ }^{10}$ Wie Detlev Kraack unterstreicht, ist diese Situation, die sich auch merklich von der Zeit davor unterschied, als Ergebnis einer Trendwende der Endsiebziger- und anfänglichen Achtzigerjahre des letzten Jahrhunderts zu begreifen, wie sie sich z. B. in der mehrbändigen Publikation von Quellen zur Geschichte Schleswig-Holsteins als Unterrichtsmaterial durch den verantwortlichen Flensburger Arbeitskreis für Stadt und Regionalforschung ab 1977, ${ }^{11}$ in der Etablierung einer eigenen Arbeitsgruppe zur Landesgeschichte im Umfeld des damaligen Kultusministeriums 1982 und im betreffenden Runderlass des Kultusministers von 1983 widerspiegelt. ${ }^{12}$ Dementsprechend schrieb etwa der Lehrplan für Gymnasien aus dem Jahr 1986 von der Unter- bis zur Oberstufe eine stattliche Reihe landesgeschichtlicher Themen verbindlich für eine Behandlung im Unterricht vor oder regte zumindest zu deren Einbeziehung als Unterrichtsgegenstand an. In der Klassenstufe 6 z. B. sollte es u. a. im Rahmen von bis zu sechs Unterrichtsstunden um Angeln, Sachsen, Wikinger, Haithabu und das Danewerk gehen, in Klasse 7 um „Freie Bauern am Beispiel Dithmarschens Geschlechter, Hemmingstedt (1500)“ oder vier bis sechs Stunden lang um die „Entstehung und Entwicklung des Herzogtums Schleswig und der Grafschaft Holstein“ mit Schau-

9 Siehe dazu Peter Gautschi, Schweizer Jugendliche und Geschichte. Befragung von 359 Luzerner Gymnasiastinnen und Gymnasiasten zum Geschichtsunterricht, 2014, https://www.phlu.ch/_Resources/Persistent/1a1dff249123d6707411767dd3c6bc2ad410a18a/2-GautschiMessmer-20150425.pdf (letzter Zugriff: 27.5.2021); Magne Angvik/Bodo von Borries (Hrsg.), Youth and History. A Comparative European Survey on Historical Consciousness and Political Attitudes among Adolescents. Vol. B: Documentation. Hamburg 1997, 143.

10 Dazu und zum Folgenden Detlev Kraack, Landes-, Regional- und Lokalgeschichte - bislang weitgehend ungenutzte Potentiale für die schulische Vermittlung von Geschichte, in: Auge/Göllnitz, Landesgeschichte (wie Anm. 2), 109-133, hier 123-127.

11 Quellen zur Geschichte Schleswig-Holsteins. 5 Bde. Hrsg. von dem Deutschen Grenzverein e.V. (Forschungsrat) und dem Landesinstitut Schleswig-Holstein für Praxis und Theorie der Schule. Kiel 1977-1989 (IPTS-Beiträge für Unterricht und Lehrerbildung, Bde. 2/14/16/19/22).

12 Kraack, Lokalgeschichte (wie Anm. 10), 124. 
enburgern, Constitutio Valdemariana (1326), Ripener Privileg (1460), „Landesherr, Räte[n], Stände[n], Ritterschaft, Realunion, Personalunion - Vom Personenverband zum institutionellen Flächenstaat“. Auch sollten in einer weiteren Unterrichtseinheit Städte mit Fernhändlern, Markt und Stadtrecht u. a. am Beispiel Lübecks behandelt und die Deutschen und ihre östlichen Nachbarn Wenden, Polen, Pruzzen angesprochen werden. ${ }^{13}$ Man gewinnt geradezu den Eindruck, dass ein landeshistorisch-mediävistisches Feuerwerk im damaligen Schulunterricht entzündet werden sollte oder konnte. Ab der 8. Klasse sollten schließlich zahlreiche neuzeitliche und zeitgeschichtliche Gegenstände der Landes- und Regionalgeschichte im Unterricht bearbeitet werden. ${ }^{14}$ Im Hinblick auf die Rolle der Landesgeschichte im Unterricht stellte der Lehrplan im Übrigen explizit und an ganz prominenter Stelle heraus: „Die Einbeziehung der Landesgeschichte soll nicht zu einer Ausweitung des behandelten Stoffes führen; Probleme der Landesgeschichte sollen vielmehr in exemplarischer Weise Fragen der allgemeinen deutschen bzw. europäischen Geschichte erhellen. [...] Nicht die bloße Rezeption, sondern die Auseinandersetzung mit historischen Inhalten ist die Aufgabe des Geschichtsunterrichts von Anfang an. Dabei ist der Gegenwartsbezug von besonderer Bedeutung. " ${ }^{15}$ Im Grunde genommen sind hiermit bereits wesentliche Motivationen zur und Gründe für eine Betrachtung landes- und regionalhistorischer Gegenstände im Geschichtsunterricht benannt, wie sie heute ganz aktuell propagiert werden. ${ }^{16}$

Innerhalb dieses Lehrhorizonts und daran anknüpfend bewegten sich auch die schleswig-holsteinischen Lehrpläne von 1997 und 2002, die verschiedene konkrete Unterrichtsinhalte aus der schleswig-holsteinischen Landes- und Regionalgeschichte in übergeordnete Themenbereiche eingebettet auflisteten. Dazu zählten etwa „Die Reformation in Schleswig-Holstein“ oder „Frühmoderner Verwaltungsstaat und Gottorfer Hofkultur“ oder „Der deutsch-dänische Konflikt bis zum Ende des Gesamtstaates 1864“ usw. ${ }^{17}$ Zudem sollte die Konkretion überregionaler Themenfelder anhand regionaler Beispiele ermöglicht werden, etwa beim Thema „Stadt“ oder „Hanse“. ${ }^{18}$ Programmatisch liest sich die Begründung hierfür im betreffenden Lehrplantext: „Der Geschichtsunterricht macht es sich zunutze, daß die Regionalgeschichte Anschauungsfeld für

13 Lehrplan Gymnasium Geschichte Klassenstufen 6-10 (Quinta bis Untersekunda). Hrsg. von dem Kultusminister des Landes Schleswig-Holstein. Kiel 1986, 6-28.

14 Lehrplan Gymnasium Geschichte Oberstufe. Hrsg. von dem Kultusminister des Landes Schleswig-Holstein. Kiel 1986,

11-27. Siehe die Tabelle bei Kraack, Lokalgeschichte (wie Anm. 10), 125.

15 Lehrplan 1986 (wie Anm. 13), 1.

16 Vgl. etwa John, Lokal- und Regionalgeschichte (wie Anm. 3), $108 \mathrm{f}$.

17 Lehrplan für die Sekundarstufe I der weiterführenden allgemeinbildenden Schulen - Hauptschule, Realschule, Gymnasium. Geschichte. Hrsg. von dem Ministerium für Bildung, Wissenschaft, Forschung und Kultur des Landes Schleswig-Holstein. Kiel/Glückstadt 1997, 21. Neben den drei genannten Themen wurden ebd. außerdem die landesgeschichtlichen Inhalte ,Die Dänen Nordschleswigs im Kaiserreich', ,Matrosen- und Arbeiteraufstand in Kiel' und ,Versailler Vertrag und die Abstimmungen in Schleswig' genannt, die in übergeordnete Themen einzubetten sind. Mittelalterliche Inhalte aus der Landesgeschichte wurden hier nicht explizit angeführt.

18 Lehrplan 1997 (wie Anm. 17), 21. 
die überregionale Geschichte ist und eine Vermittlerrolle zwischen der Geschichte des ,kleinen Raumes' und der nationalen Geschichte spielen kann. " ${ }^{19}$ Und weiter hieß es darin: „Die Landesgeschichte läßt sich vor Ort und in der Region aufspüren und bietet die Möglichkeit zu lebensweltgebundenem und zu exemplarischem Unterricht. Mit der Hinwendung zu den Nahräumen - Landschaft, Region, Stadt, Stadtteil, Dorf - ergeben sich zudem die didaktischen Vorzüge der Überschaubarkeit, Konkretheit, Authentizität und Detailtreue. Die Arbeit am überschaubaren Raum kann mit Hilfe entsprechender Lernverfahren und vielfältiger Materialien methodenorientiertes und selbständiges Lernen fördern. “ ${ }^{20}$ Deutlich spiegelt sich in Wortwahl und Intention die seinerzeitige Debatte über den Perspektiven- und Methodenwandel von der klassischen Landes- zur jüngeren Regionalgeschichte wider. ${ }^{21}$

Demgegenüber stellen sich nun freilich die schleswig-holsteinischen Fachanforderungen für das Unterrichtsfach Geschichte vom Jahr 2016 ganz anders dar: Die Fachanforderungen plädieren zwar dafür, „[d]ie Regional- und Landesgeschichte Schleswig-Holsteins [...] angemessen zu integrieren“. ${ }^{22}$ Auf die zuvor in den Lehrplänen vorhandenen ausführlichen Angaben und Handreichungen zur Thematisierung von Landes- und Regionalgeschichte wird jetzt allerdings unter dem Vorzeichen der gewünschten und ganz zentral ins Auge gefassten Kompetenzorientierung ${ }^{23}$ weitgehend verzichtet. Konkret werden lediglich für den Einführungsjahrgang (10. oder 11. Klasse) für das zweite Halbjahr (E2) zum Bereich „Begegnungen von Kulturen - Konfrontation, Abgrenzung oder Integration?“ für den historischen Inhalt „Die Deutschen und ihre Nachbarn“ der Aspekt „Deutsche und Dänen - Vorbild für ein zusammenwachsendes Europa“ und für die 11. bzw. 12. Jahrgangsstufe (Qualifikationsphase Q 1.2) die problemorientierte Fragestellung „Dänemark - neue Identität nach der militärischen Niederlage 1864?" als regionalhistorisch relevante Aspekte angeführt. ${ }^{24}$ Mit dieser veränderten Ausrichtung steht Schleswig-Holstein natürlich nicht für sich, sondern liegt ganz im Trend der aktuellen deutschen Lehrpläne, in deren Inhalten und Zielsetzungen Stephan Laux nicht nur eine Erosion der Landesgeschichte allgemein erkennen möchte, sondern

19 Lehrplan 1997 (wie Anm. 17), 20.

20 Ebd., 21.

21 Vgl. dazu allgemein Matthias Werner, Zur Geschichte des Faches, in: Werner Freitag/Michael Kißener/Christine Reinle/ Sabine Ullmann (Hrsg.), Handbuch Landesgeschichte. Berlin/Boston 2018, 3-23, hier 12f.; Peter Claus Hartmann, Landesund Regionalgeschichte in Europa in Vergangenheit, Gegenwart und Zukunft, in: BlldtLG 148, 2012, 277-286.; Werner Freitag, Landesgeschichte als Synthese - Regionalgeschichte als Methode?, in: WestF 54, 2004, 291-305; Reinhard Stauber, Regionalgeschichte versus Landesgeschichte? Entwicklung und Bewertung von Konzepten der Erforschung von „Geschichte in kleinen Räumen", in: Geschichte und Region/Storia e regione 3, 1994, 227-260.

22 Fachanforderungen Geschichte. Allgemein bildende Schulen. Sekundarstufe I und II, hrsg. vom Ministerium für Schule und Berufsbildung des Landes Schleswig-Holstein. Kiel 2016, 20.

23 Ebd., 8 (2.1 Kompetenzorientierung), 15-19, aufzuschlüsseln nach Wahrnehmungs-, Erschließungs-, Sachurteils- sowie Orientierungskompetenz.

24 Ebd., 28 bzw. 31. 
auch „eine Erosion der verbliebenen regionalgeschichtlichen Bestandteile“.25 „In einer ganzen Reihe von Lehrplänen werden regionale Aspekte nach wie vor für möglich erklärt und auch mit einer gewissen Wertschätzung bedacht“ - wie es eben auch in Schleswig-Holstein der Fall ist. „Dabei handelt es sich aber nie um Landesgeschichte als konsistente thematische und didaktische Komponente, sondern meist um bescheidene Entlehnungen regionaler oder lokaler Gegenstände. Sie erscheint im Wesentlichen in Form von wähl- bzw. austauschbaren Stoffpartikeln, die den mit dem Fach verbundenen ,Bildungszielen` durchweg untergeordnet werden." ${ }^{26}$

Für die einen bietet die seit 2016 veränderte Situation durchaus verständlichen Anlass zu großem Wehklagen. Der vorne schon zitierte Detlev Kraack etwa, seines Zeichens aktiver Gymnasiallehrer in Schleswig-Holstein für die Fächer Geschichte und Latein und gleichzeitig überaus umtriebiger Landes- und Regionalhistoriker, schreibt dazu aus seiner Sicht: „So werden regional- und landesgeschichtlich interessierte und sozialisierte Kolleginnen und Kollegen konkrete Vorschläge zur Einbettung landes- und regionalgeschichtlicher Inhalte und Gegenstände in den Unterricht schmerzlich vermissen. Stattdessen propagieren die Fachanforderungen wortreich den Paradigmenwechsel zu einer ganz gezielten Kompetenz-, Themen- und Problemorientierung. Das muss lokal- und regionalgeschichtliche Zugangsweisen nicht a priori ausschließen. Es birgt indes ganz offensichtlich die Gefahr, dass diese hinfort nicht mehr gleichsam selbstverständlich zur Anwendung kommen. Fachfremd Geschichte unterrichtende Kolleginnen und Kollegen werden überdies lieber durch Schulbücher und übergeordnete Themenhefte ,abgesicherte' Gegenstände vor den Schülerinnen und Schülern ausbreiten, als sich in neue, bislang völlig unbekannte Gegenstände einzuarbeiten und sich in der Nahumgebung der Schülerinnen und Schüler die Blöße von Wissenslücken zu geben. “27 Ganz abgesehen davon, dass die alten Lehrpläne letzteres Problem der möglichen Bloßstellung landes- und regionalhistorischer Wissensdefizite auch nicht ohne Weiteres gelöst oder auch nur in den Griff bekommen hätten und umgekehrt manches derzeit im Land im Gebrauch befindliche Schulbuch durchaus ,abgesicherte‘ Gegenstände der Landesund Regionalgeschichte für den Lehrgebrauch im Schulunterricht anbietet, ${ }^{28}$ konzediert Kraack immerhin, die Fachanforderungen würden die besondere Eignung regional- und landeshistorischer Inhalte zur Erstellung einer fundierten ,Narratio unterstreichen, und verweist auf die beiden genannten Konkretisierungen zur deutsch-dänischen Geschichte,

25 Stephan Laux, Clio und Curriculum. Lehrpläne der Sekundarstufen I und II im bundesdeutschen Vergleich und Perspektiven der Landesgeschichte, in: Auge/Göllnitz (Hrsg.), Landesgeschichte (wie Anm. 2), 17-40, hier 31 in Fortführung von bzw. Auseinandersetzung mit Horst Kuss, Landesgeschichte oder Regionalgeschichte? Über den Umgang von geschichtswissenschaftlicher Theorie, geschichtsdidaktischer Konzeption und praktischer Anwendung im Unterricht, in: GWU 51, 2000, 388-405, hier 395-401.

26 Die Zitate stammen aus Laux, Clio (wie Anm. 25), 31. Siehe auch ebd., 35-37 „Ein Problem nicht nur der Landesgeschichte: Lehrpläne zwischen Über- und Unterregulierung".

27 Kraack, Lokalgeschichte (wie Anm. 10), 131.

28 Siehe dazu das noch Folgende und nunmehr die landesübergreifende Sichtung von Martin Göllnitz/Tomke Jordan, „Heimatgeschichte", "Vertiefung" oder "Projekt". Landes-und Regionalgeschichte im Schulbuch am Beispiel der Verlage Schöningh, Schroedel und Westermann, in: HessJBLG 70, 2020, 301-318. 
die im weiteren Sinn regional- und landeshistorische Themenfelder darstellen. ${ }^{29}$ Doch die Schlussfolgerung, die Kraack aus seiner schulischen Praxis heraus zieht, klingt ernüchternd: Wie diese Anforderungen konkret mit Leben gefüllt werden, „bleibt den Kolleginnen und Kollegen vor Ort bzw. den schulinternen Absprachen im Rahmen der Fachkonferenzen sowie der Fixierung und Fortschreibung in ,Schulinternen Fachcurricula' vorbehalten. Dass hier die Fachkonferenzen vor Ort gefragt sind, lässt die Sache in dem Augenblick problematisch werden, wo fachfremd unterrichtende Lehrkräfte oder solche ohne Kenntnis der lokalen Unterrichtspotenziale verantwortlich Kompetenz entwickeln und in der unterrichtlichen Welt umsetzen sollen. “30

Den anderen dagegen erscheint der aktuelle Ansatz der Fachanforderungen demgegenüber gerade als bisher so nicht vorhandene Chance für den Einsatz regional- und landeshistorischer Gegenstände im Schulunterricht. Angesichts eines anscheinend vergleichsweise geringen Interesses der Schülerinnen und Schüler an Landesgeschichte, was mit - auf ihre Art sicher hinterfragbaren - Zahlen aus Baden-Württemberg und empirischen eigenen Erfahrungen untermauert wird, und unter Betonung der Besonderheit wie zugleich Herausforderung des "didaktische[n] Vorzug[s] der Nähe“, ${ }^{31}$ birgt für Rolf Schulte, den ehemaligen Studienleiter für Geschichte am Institut für Qualitätsentwicklung an Schulen in Schleswig-Holstein (IQSH) und langjährigen Lehrbeauftragten für Fachdidaktik am Historischen Seminar der Christian-Albrechts-Universität zu Kiel (CAU), der Nahraum „ein großes Lernpotential, wenn er nicht als Feld neben allgemeiner Geschichte, sondern wenn das Allgemeine im Konkreten deutlich wird. Regionale geschichtliche Beispiele sind dann exemplarische Fälle für umfassende europäische und globale Entwicklungen, wenn sie das jeweils Typische eines Vorgangs widerspiegeln, da derartige Prozesse oft gleichen Ursachen zugrunde liegen und in ähnlichen Formen verlaufen. “32 Nur am Rande sei an dieser Stelle daran erinnert, dass die bloß exemplifizierende Rolle der Landes- und Regionalgeschichte von Laux moniert worden ist. Im Hinblick auf die Fachanforderungen äußert sich Schulte des Weiteren ganz optimistisch: „Derartig offen formulierte Fachanforderungen, die sich durch geringe Reglementierungsdichte auszeichnen, erlauben je nach Lehrkraft einen stark regionaloder landesgeschichtlich ausgerichteten Unterricht, wie er zuvor kaum möglich war, denn zahlreiche Inhalte lassen sich oft wirkungsvoll regional- oder landesgeschichtlich

29 Kraack, Lokalgeschichte (wie Anm. 10), mit Fachanforderungen (wie Anm. 22), 13: „Narrative Kompetenz bedeutet in diesem Zusammenhang, dass die Schülerinnen und Schüler lernen, ihre persönlichen geschichtlichen Vorkenntnisse und Voreinstellungen in eine methodisch fundierte Narration zu übertragen. Hierfür eignen sich besonders regional- und landesgeschichtliche Inhalte, die im schulinternen Fachcurriculum ausgewiesen werden." - Vgl. zu den Fachcurricula die Fachanforderungen (wie Anm. 22), 34

30 Kraack, Lokalgeschichte (wie Anm. 10), 131.

31 Rolf Schulte, Landes- oder Regionalgeschichte in der Schule?, in: Auge/Göllnitz, Landesgeschichte (wie Anm. 2), 93-108, hier 101: „Diese Feststellung gilt allerdings nur, solange der didaktische Vorzug der Nähe genutzt wird. Für die Schülerschaft im Großraum Hamburg liegen diese beiden Städte (sc. Rendsburg und Flensburg) mental ebenso weit weg wie Rostock in Mecklenburg-Vorpommern oder Braunschweig in Niedersachsen."

32 Ebd., mit Verweis u. a. auf Wolfgang Emer, Die Region als didaktischer Ansatz im Geschichtsunterricht. Standortbestimmung und praktische Umsetzung, in: Demokratische Geschichte 21, 2010, 209-230. 
exemplifizieren. “" ${ }^{33} \mathrm{Ob}$ angesichts des breiten Raums, den die früheren Lehrpläne der Landes- und Regionalgeschichte, wie gezeigt, ganz konkret einräumten, in den nunmehrigen Fachanforderungen ein zuvor nicht dagewesenes Potential für die Vermittlung von Landes- und Regionalgeschichte vorhanden ist, wie Schulte meint, ist sicher Ermessenssache, die sich gut und gern gleichsam in die entgegengesetzte Richtung deuten ließe. Allgemein und auch in Schleswig-Holstein liegt der Schwerpunkt der Stoffauswahl mit nach wie vor steigender Tendenz jedenfalls eher auf globalen, europäischen oder zumindest nationalen Themen, wie Bernd Schönemann bereits 2008 feststellte. ${ }^{34}$ Zudem ist die vorherrschende Prädisposition der aktiven Lehrer*innenschaft in Rechnung zu stellen, die sich zumindest nach meinem Eindruck oftmals durch eine worin auch immer begründet liegende Distanz zum landes- oder regionalhistorisch ausgerichteten Unterricht auszeichnet. ${ }^{35}$ Insofern ist es nur zu ehrlich, wenn Schulte eingesteht: „Jedoch schreiben (die Fachanforderungen) ein derartig orientiertes geschichtliches Lernen nicht mehr verbindlich vor: Eine Lehrkraft kann also auch die Thematisierung von Region oder Land marginalisieren." ${ }^{36}$ Und wenn das Verhältnis der Lehrer*innenschaft zur Regionalgeschichte weiterhin so distanziert bleibt, wie es aktuell nach meinem Dafürhalten leider überwiegt, dürfte das doch die weithin wahrscheinlichere Option darstellen.

Das soll nun aber ganz und gar nicht heißen, dass es regionalhistorische Hoffnungsschimmer, wie zitierter Schulte selbst während seiner aktiven Dienstzeit einen darstellte und genannter Kraack nach wie vor als einer wirkt, nicht doch auch in der aktiven Lehrerschaft Schleswig-Holsteins gibt - und solche herausragenden Beispiele der Activitas können und möchten im wahrsten Sinne des Wortes Schule machen. So plädiert etwa der Gymnasiallehrer Volker Gaul gemeinsam mit Sebastian Barsch, Inhaber der Kieler Professur für Didaktik der Geschichte, mit Nachdruck für einen Einsatz regionalhistorischer Themen als ,Good Practice für subjektorientiertes Lernen in Schulen. ${ }^{37}$ Dabei gehe es nicht, was mittlerweile allgemein klar geworden sein sollte, um die Stiftung naiver Identitäten oder auch nicht um eine falsche Betonung einer besonderen Dignität des Lerngegenstandes Regionalgeschichte - wohlgemerkt meinte auch Laux nicht Dignität, sondern Eigenwert, wie ihn National- oder Globalgeschichte

\footnotetext{
33 Schulte, Landes- oder Regionalgeschichte (wie Anm. 31), 97.

34 Oliver Auge/Martin Göllnitz, Wozu Landes- und Regionalgeschichte an der Schule? Einige Vorbemerkungen und Überlegungen, in: Dies., Landesgeschichte (wie Anm. 2), 1-16, hier 1 mit Verweis auf den Sektionsbericht zum Historikertag 2008: Martin Schlutow/Tim Völkering, Tagungsbericht: HT 2008: Geschichtsvermittlung durch Landes- und Regionalgeschichte. Ein deutsch-tschechischer Vergleich, 30.9.-3.10.2008 Dresden, in: H-Soz-Kult, 24.10.2008, www.hsozkult.de/conferencereport/ id/tagungsberichte-2297 (letzter Zugriff: 27.5.2021).

35 Dazu Auge/Göllnitz, Wozu (wie Anm. 34), 4. - Vgl. dazu Johannes Dillinger, Aspekte der Vermittlung von Landesgeschichte an der Universität, in: Ders. (Hrsg.), Die Vermittlung von Landesgeschichte. Beiträge zur Praxis der historischen Didaktik. Heidelberg u. a. 2010, 72-85.

36 Schulte, Landes- oder Regionalgeschichte (wie Anm. 31), 97

37 Sebastian Barsch/Volker Gaul, Regionalgeschichte forschend entdecken - eine Herausforderung für Lehrkräfte, eine Chance für subjektorientiertes historisches Lernen in Schulen, in: Auge/Göllnitz, Landesgeschichte (wie Anm. 2), 71-92, hier 76f.
} 
gewissermaßen unhinterfragt für sich beanspruchen zu dürfen. Vielmehr erscheine der Einsatz der Regionalgeschichte im Schulunterricht aus rein pragmatischen Aspekten, etwa wegen der besseren Erreichbarkeit von Archiven, außerschulischen Lernorten und Zeitzeugen, wegen der gegebenen Realanschauung - hierzu ließe sich schlaglichtartig das Motto „Überall ist Regionalgeschichte“ zitieren ${ }^{38}$-, aufgrund der gerade hier sehr gut möglichen Teilhabe am Prozess der historischen ,Narratio“ sowie mittels vorhandener geschichtskultureller Realerfahrungen besonders sinnvoll und vielversprechend. In Fortführung von Gedanken Bernd Schönemanns - und letztlich ganz ähnlich wie schon die eingangs zitierte Einleitung zur Göppinger regionalhistorischen Quellen- und Textsammlung - halten beide Verfasser das im heutigen Schulunterricht stark gemachte Forschende Lernen gerade im regionalhistorischen Kontext für sehr gut realisierbar. ${ }^{39}$ Schönemann hatte hierzu bereits 2010 geschrieben: „Der zu einem eigenständigen Typus aufgewertete erkundende Geschichtsunterricht [...], der das Verlassen der Schule und das Aufsuchen eines außerschulischen Lernortes ebenso zwingend vorsieht wie die Begegnung mit Originalquellen, ist als Lehr-Lern-Konzept geradezu prädestiniert für regionalgeschichtliche Themen und ,geschichtskulturelle Realerfahrungen“ im regionalen Umfeld. “ ${ }^{40}$ Das Autorenduo Barsch und Gaul veranschaulicht die von ihm unterstrichene ausgezeichnete Qualität regionalhistorisch ausgerichteten Geschichtsunterrichts, wie sie ja auch in den Fachanforderungen explizit benannt ist, ${ }^{41}$ am Praxisbeispiel des von der Körber-Stiftung organisierten Geschichtswettbewerbs des Bundespräsidenten, der regelmäßig die didaktischen Ziele der Subjektorientierung und des Forschenden Lernens unter dem Dach eines regionalhistorischen Bezugs zusammenführt. ${ }^{42}$ „Der Geschichtswettbewerb stellt dafür einen extrinsischen Motivationsimpuls dar, der eine dauerhafte intrinsische Motivation initiieren soll. “43 Konkret führen sie drei in diesem Kontext prämierte schleswig-holsteinische Beispiele vor Augen: einen Klassenbeitrag zur Hexenverfolgung in Dithmarschen, einen klassenübergreifenden Gruppenbeitrag über einen Fall von Sodomie in Hennstedt im 17. Jahrhundert sowie einen Einzelbeitrag, der nach Wahrheit oder Mythos bezüglich der sog. Lutherglocke von Hemmingstedt fragte. ${ }^{44}$

Tatsächlich sind die praktischen Möglichkeiten des Einsatzes regionalhistorischer Zugänge zur Vergangenheit im Schulunterricht anhand dieser Preisarbeiten im positiven Sinn frappierend ${ }^{45}$ Sie stellen aber, was betont werden muss, bislang sicher doch eine

38 Vgl. dazu Oliver Auge, Was meint und macht Regionalgeschichte an der CAU zu Kiel?, in: Mitteilungen der Gesellschaft für Schleswig-Holsteinische Geschichte 90, 2016, 7-18, hier 14.

39 Barsch/Gaul, Regionalgeschichte (wie Anm. 37), 77.

40 Bernd Schönemann, Lernpotentiale der Regionalgeschichte, in: geschichte für heute. zeitschrift für historisch-politische bildung 3.2, 2010, 5-16, hier 12 (Hervorhebung im Original).

41 Fachanforderungen (wie Anm. 22), 13.

42 Barsch/Gaul, Regionalgeschichte (wie Anm. 37), $79 f$.

43 Zitat aus ebd., 80.

44 Ebd., 80-90.

45 Vgl. dazu allgemein auch John, Lokal- und Regionalgeschichte (wie Anm. 3), 67-71. 
Ausnahme im praktischen Schulunterricht dar, was allein schon darin begründet liegt, dass der Geschichtswettbewerb immer nur im zweijährigen Abstand ausgeschrieben wird, also nicht turnusmäßig in jedem gleichen Jahrgang durchgeführt werden kann. ${ }^{46}$ Immerhin verdichtet sich die Schulwettbewerbslandschaft in Schleswig-Holstein weiter, was sich nur positiv auf die stärkere Implementierung Forschenden Lernens in der Unterrichtsgestaltung auswirken wird. So schrieb die Bürgerstiftung Schleswig-Holsteinische Gedenkstätten 2019/20 erstmalig ebenfalls einen Schüler*innenwettbewerb aus. Sein Thema lautete ,EURE Erinnerung. Dem Betätigungsfeld der Bürgerstiftung entsprechend sollte die Auseinandersetzung mit der NS-Gewaltherrschaft in SchleswigHolstein dabei im Mittelpunkt stehen. ${ }^{47}$ Die von Barsch und Gaul vorgestellten Themen rekurrieren immerhin auf frühneuzeitliche Zusammenhänge und Quellen, was angesichts der Prädominanz der Zeitgeschichte nach 1945 im Schulunterricht ${ }^{48}$ höchst erfreulich und ermutigend ist. Sie lassen die mittelalterliche Epoche, um die es uns hier besonders gehen soll, allerdings ebenfalls links liegen.

\section{Die Potentiale: Regionalgeschichte zum Mittelalter - ein Thema für Schulen in Schleswig-Holstein!}

Es wurde bereits auf den Hinweis in den aktuellen Fachanforderungen aufmerksam gemacht, dass die Regional- und Landesgeschichte Schleswig-Holsteins angemessen in den Geschichtsunterricht integriert werden soll. ${ }^{49}$ Und es wurde auch schon vermerkt, dass im weiteren Sinn zwei Themenfelder konkret auf die Landes- und Regionalgeschichte Schleswig-Holsteins verweisen: „Deutsche und Dänen - Vorbild für ein zusammenwachsendes Europa“ und „Dänemark - neue Identität nach der Niederlage von 1864 “. ${ }^{50}$ Letzterer Themenbereich ist eindeutig in der neueren Geschichte angesiedelt, ersterer auch eher in selbiger und der Zeitgeschichte, was angesichts der in den Fachanforderungen direkt propagierten Prädominanz der Zeitgeschichte im Geschichtsunterricht nicht verwundert. Indes bieten sich innerhalb der Fachanforderungen, die bewusst auf ein Zuviel an Regulierung verzichten ${ }^{51}$, durchaus einige Potentiale für eine Vermittlung auch mittelalterlicher regionalhistorischer Inhalte im modernen Schulunterricht.

\footnotetext{
46 Siehe die Angaben zum Geschichtswettbewerb unter https://www.koerber-stiftung.de/geschichtswettbewerb/portraet (letzter Zugriff: 1.4.2021).

47 Siehe die Informationen unter https://gedenkstaetten-sh.de/eure-erinnerung--erster-schuelerwettbewerb-der-buergerstiftung-mit-grosser-resonanz-und-beeindruckenden-ergebnissen (letzter Zugriff: 27.5.2021).

48 Siehe dazu die Fachanforderungen (wie Anm. 22), 20: „Die Zeitgeschichte nach 1945 muss mindestens ein Viertel der insgesamt zur Verfügung stehenden Unterrichtszeit im Fach Geschichte in der Sekundarstufe I in Anspruch nehmen."

49 Siehe nochmals ebd.

50 Siehe ebenfalls nochmals ebd., 28 und 31

51 Ebd., 7.
} 
Im ersten chronologisch-genetisch strukturierten Durchlauf ist immerhin noch ein eigener Themenblock für das Mittelalter allgemein als ein Halbjahresthema vorgesehen: „Das Mittelalter - eine finstere Zeit?“, wobei speziell auf Lebens- und Herrschaftsformen und das Verhältnis von Juden, Christen und Muslimen im Mittelalter als historische Inhalte eingegangen werden soll. ${ }^{52}$ Rolf Schulte führt in seinem schon näher berührten Beitrag ,Landes- oder Regionalgeschichte in der Schule?‘ aus, wie anhand des regionalhistorischen Beispiels des holsteinischen Grafen Adolf III. von Schauenburg allgemeine Ereignisse, Abläufe und Biografien der Kreuzzugsgeschichte als Kulminationspunkt jüdisch-christlich-muslimischer Begegnung im Mittelalter reibungslos in der schulischen Praxis vermittelt werden können. ${ }^{53}$ Nicht von ungefähr existiert genau für dieses Fallbeispiel im ersten Band des Schulbuchs Geschichte entdecken, das genannter Schulte gemeinsam mit Benjamin Stello herausgegeben hat, eine passende regionalhistorische Handreichung für den Unterricht: „Ein Kreuzritter aus Holstein im Orient: Graf Adolf III.“, die mit einem nahezu einseitigen Quellenauszug aus der Chronik des Lübecker Abts Arnold sowie zwei Abbildungen als vielversprechende Arbeitsgrundlage versehen ist. ${ }^{54}$

Daneben finden sich in demselben Schulbuch immer wieder regionalhistorische Fingerzeige eingestreut, etwa wenn aktuelle Mittelalter-Märkte in Schleswig-Holstein aufgelistet sind und auf derselben Seite die Aufgabe gestellt wird, am jeweiligen Wohnort die Gebäude und Straßennamen ausfindig zu machen, die auf das Mittelalter verweisen. ${ }^{55}$ Zur Behandlung der mittelalterlichen Stadt- und Handelsgeschichte wird stark auf das naheliegende Beispiel Lübeck zurückgegriffen. ${ }^{56}$ Im Folgeband, der sich epochenmäßig von der frühen Neuzeit bis zum Ende des 19. Jahrhunderts erstreckt, machen schleswig-holsteinisch-regionalhistorische Beispiele acht Prozent des gesamten Buchinhalts aus.$^{57}$ Einen noch stärkeren regionalhistorischen Akzent setzt indes das Schulbuch „Das waren Zeiten“, das programmatisch schon auf den Titelbildern seiner für uns hier besonders interessanten, da das Mittelalter in den Blick nehmenden Bände 1 und 2 einmal das Nydam-Boot und zum anderen das Wappen der Gottorfer Herzöge im Schleswiger Dom präsentiert und über eigene Themenblöcke, Abbildungen, Materialien und Lern- und Exkursionstipps eine große Bandbreite regionalhistorischer Bezüge zum Mittelalter (und darüber hinaus natürlich) bietet. ${ }^{58}$ Die prononcierte Ausrichtung auf regionalhistorische Bedürfnisse spiegelt allein die Tatsache wider, dass der verantwortliche

\footnotetext{
52 Fachanforderungen (wie Anm. 22), 20.

53 Schulte, Landes- oder Regionalgeschichte (wie Anm. 31), 105-107.

54 Rolf Schulte/Benjamin Stello (Hrsg.), Geschichte entdecken, Bd. 1: Von der Ur- und Frühgeschichte zum Mittelalter. Schleswig-Holstein. Bamberg 2017, $212 \mathrm{f}$.

55 Ebd., 195.

56 Ebd., 178-185.

57 Benjamin Stello (Hrsg.), Geschichte entdecken, Bd. 2: Von der Frühen Neuzeit bis ins 19. Jahrhundert. Schleswig-Holstein. Bamberg 2017. Ich verdanke die zahlenmäßige Auswertung Frau Tomke Jordan, Kiel.
}

58 Dieter Brückner/Harald Focke (Hrsg.), Das waren Zeiten, Bd. 1: Von den ersten Menschen bis zum Mittelalter. SchleswigHolstein. Bamberg 2009 und Dies. (Hrsg.), Das waren Zeiten, Bd. 2: Von der Hansezeit bis zur Industrialisierung. Bamberg 2010. 
Verlag C.C.Buchner auf der Produktinternetseite des Schulbuchs die „Regionalbezüge in unserem Geschichtsbuch" ausweist, was für meine auswertende Arbeit sehr hilfreich gewesen ist. ${ }^{59}$ Gerade mit diesem Lernmittel kann das erwähnte Postulat der Fachanforderungen, die Regional- und Landesgeschichte Schleswig-Holsteins angemessen im Unterricht zu berücksichtigen, vielseitig und eigentlich problemlos eingelöst werden. Das gilt im Prinzip selbstredend zumal für das Mittelalter, wenn denn in der Unterrichtspraxis genügend Zeit für und ausreichendes Interesse an dieser Epoche vorhanden ist.

Nach dem ersten überwiegend chronologisch strukturierten Durchgang durch die Geschichte in der Sekundarstufe I erfolgt der Unterricht nach den geltenden Fachanforderungen in Sekundarstufe II unter Aufhebung der strengen chronologischen Ordnung nunmehr themenzentriert. ${ }^{60}$ Auf den ersten Blick ist das angedachte Unterrichtsmodell in der neueren und neuesten Geschichte verankert, wenn im zweiten Teil des Einführungsjahrgangs E2 „Begegnungen von Kulturen“ die Themenblöcke Migration in der Geschichte und Imperialismus sowie im dritten Teil E3 „Wandel von Wirtschaft und Gesellschaft“ die Bereiche Feudalismus, Industrialisierung und Globalisierung als historische Aspekte behandelt werden sollen. ${ }^{61}$ Allerdings bietet die angesprochene Aufhebung der strengen Chronologie ihrerseits zumindest die Option, bei den einzelnen neuzeitlichen oder zeithistorischen Themen doch auf mittelalterliche Bezüge oder gar Ursprünge der Phänomene und weiteren Entwicklungen in der Neuzeit zu verweisen, nicht zuletzt um die longue durée so mancher geschichtlicher Vorgänge und Linien zu verdeutlichen. Stets nur auf die neuzeitliche Seite der Phänomene zu schauen, verfälscht sie gar in ihrer historischen Genese und erschwert oder verflacht zumindest insgesamt deren Verständnis. So ist Migration natürlich ein Kontinuum in der Geschichte der Menschen, seit diese in der Urzeit ihr Leben als wandernde Jäger und Sammler fristeten. Zahlreiche Beispiele von Migration lassen sich natürlich auch zum regionalhistorischen Mittelalter beibringen, wovon die Ostsiedlung sicher ein bekannteres, aber bei weitem nicht das einzige ist. ${ }^{62}$ Das weitere Thema Feudalismus bietet sich per se an, um an dessen Ursprünge im Mittelalter zu erinnern und nicht allein seine neuzeitliche Entwicklung im Rahmen der hierzulande besonders stark ausgeprägten Gutswirtschaft ${ }^{63}$ in den Blick zu nehmen. Hier ist der Bezug zum regionalen Mittelalter sicher sogar am offensichtlichsten. ${ }^{64}$ Doch auch die Themenfelder der Industrialisierung

59 Siehe unter "weitere Serviceangebote” auf der jeweiligen Produktinternetseite bei C.C.Buchner für Bd. 1: https://www. ccbuchner.de/titel-7-7/von_den_ersten_menschen_bis_zum_mittelalter-1706/, Bd. 2: https://www.ccbuchner.de/titel-7-7/ von_der_hansezeit_bis_zur_industrialisierung-1707/ und Bd. 3: https://www.ccbuchner.de/titel-7-7/deutschland_europa_ und_die_welt_von_1871_bis_zur_gegenwart-1708/ (letzte Zugriffe: 27.5.2021).

60 Fachanforderungen (wie Anm. 22), $26 f$.

61 Ebd., $28 f$.

62 Siehe zum Thema z. B. Dirk Meier, Menschen in Bewegung. Schleswig-Holstein als Ein- und Auswanderungsland von der Prähistorie bis zur Gegenwart. Heide 2017, hier 98-103 zur Ostsiedlung.

63 Statt vieler siehe z. B. Michael North, Die frühneuzeitliche Gutswirtschaft in Schleswig-Holstein, in: BlldtLG 126, 1990, 223-242.

64 Siehe etwa kurzgefasst Christian Degn, Schleswig-Holstein - eine Landesgeschichte. Historischer Atlas. Neumünster 1994, 52f., mit vorzüglicher Quelleninterpretation. 
und Globalisierung lassen sich zum Teil durchaus ebenfalls im Mittelalter verorten, wenn man z. B. die wichtige Rolle der Mühlen als frühe Gewerbe- und Technikzentren in Schleswig-Holstein ${ }^{65}$ oder aber die laufenden Diskussionen um die Rolle der Hanse im Rahmen der sog. Protoglobalisierung ${ }^{66}$ im Schulunterricht miteinbezieht. Wenn in diesem Kontext dann auch konkret z. B. die Auswirkungen von Industrialisierung und Globalisierung auf Menschen, Umwelt und Klima thematisiert werden, was Schülerinnen und Schüler aktuell selbst im Rahmen der ,Fridays for Future'-Proteste aktiv und öffentlichkeitswirksam zur Sprache bringen, dann kann dieses Thema ebenfalls wie selbstverständlich im regionalen Mittelalter verortet werden. Bereits die genannten Schulbücher zur Sekundarstufe I gehen beide sogar auf verhältnismäßig breitem Raum auf die „Grote Mandränke“ von 1362 und die Sturmfluten als „Begleiter" der Geschichte Schleswig-Holsteins ein. ${ }^{67}$ Interessanter Weise schlägt auch Anke John „Sündfluten und Jahrhunderthochwasser“ im unterrichtspraktischen Teil ihrer Monografie zu Lokal- und Regionalgeschichte im Geschichtsunterricht als ein mögliches, vielversprechendes Thema vor. ${ }^{68}$

\section{Regionalgeschichte und das Mittelalter im schleswig-holsteinischen Schul- unterricht: Ein kurzer optimistischer Ausblick}

Eine in meinen Lehrveranstaltungen regelmäßig durchgeführte Befragung der Studierenden zeigt, dass das Gros der Schüler*innen in den Oberstufen heutiger schleswig-holsteinischer Schulen bei einem zwei- bis dreistündigen Fachunterricht weitgehend ohne regionale Bezüge im Geschichtsunterricht auskommen muss. Das wirkt sich wiederum schwerwiegend nachteilig auf das Studienverhalten der daraus hervorgehenden Geschichtsstudierenden aus, zumindest was ihre Bereitschaft zur Kontaktaufnahme mit der Regionalgeschichte anbelangt. Die große Mehrzahl von ihnen kommt mit schleswig-holsteinischer Landes- und Regionalgeschichte oft erstmalig und im Rahmen des Studiencurriculums dann regelmäßig auch lediglich in homöopathischer Dosis in Berührung. ${ }^{69}$

Demgegenüber steht das optimistische Plädoyer eines Befürworters der derzeit geltenden Fachanforderungen, Rolf Schulte: „Ein regional- und landesgeschichtlich orientierter Unterricht bei Berücksichtigung der Schüler- und Subjektorientierung lässt sich methodisch in wenig aufwändiger Form in aufgabenorientierte und damit stärker gesteu-

65 Uwe Karstens/Wolfgang Kuhlmann, Die Wind- und Wassermühlen in Schleswig-Holstein und Hamburg. Kiel 2017 (Beiträge zur Denkmalpflege in Schleswig-Holstein, Bd. 7), 22-29 zum Mittelalter.

66 Rolf Hammel-Kiesow , Herren der Hanse", ökonomische Netzwerke und Proto-Globalisierung. Das Bild von der Hanse im frühen 21. Jahrhundert, in: Thomas Schilp/Barbara Weltzel (Hrsg.), Dortmund und die Hanse. Fernhandel und Kulturtransfer. Bielefeld 2012 (Dortmunder Mittelalter-Forschungen, Bd. 15), 17-31, hier 25-27; Ders., Europäische Union, Globalisierung und Hanse. Überlegungen zur aktuellen Vereinnahmung eines historischen Phänomens, in: Hansische Geschichtsblätter 125, 2007, 9-30.

67 Schulte/Stello, Geschichte entdecken, Bd. 1 (wie Anm. 54), 188f.; Brückner/Focke, Das waren Zeiten, Bd. 2 (wie Anm. 58), $26 f$.

68 John, Lokal- und Regionalgeschichte (wie Anm. 3), 158-168.

69 Auge/Göllnitz, Wozu (wie Anm. 34), 4. 
erte Unterrichtsformen integrieren. Er bietet aber sein innovatives Potential besonders in offenen Unterrichtsformen, die entdeckendes, forschendes und handlungsorientiertes Lernen in Projekten und an außerschulischen Lernorten fördern. Ein derartiger Geschichtsunterricht mit klarer didaktischer Zuspitzung eröffnet neben einigen Schwächen vielfache Chancen, historisches Denken zu fördern [...]. " 70

In der Tat bieten die Fachanforderungen Geschichte mit ihrer vergleichsweise großen Offenheit und ihrer Betonung der besonderen Rolle des regionalhistorischen Zugriffs für forschendes und handlungsorientiertes Lernen in ihrer Grunddisposition ein großes Potential für einen regionalhistorisch verankerten Geschichtsunterricht - und zwar für beide Sekundarstufen und nicht nur für die neuere und Zeitgeschichte, sondern eben auch für das Mittelalter. Gerade die übergreifenden, von der Chronologie gelösten Themenblöcke können im Prinzip auch sehr schön für Rückgriffe auf regionalhistorische Beispiele aus dem Mittelalter genutzt werden.

Gute Grundlagen wären also prinzipiell gelegt. Doch die Praxis sieht offensichtlich landauf landab regelmäßig anders aus. Durch eine engere Vernetzung der Arbeit der Kieler Professur für Regionalgeschichte mit dem Lehrauftrag der Schulen, wozu etwa durch den eingangs zitierten Tagungsband ein kleiner Schritt gemacht wurde und wozu auch das Engagement der Abteilung im Rahmen des Programms der Schleswig-Holsteinischen Universitätsgesellschaft „Uni kommt zur Schule“71 ihren gewissen fortlaufenden Beitrag leistet, und durch eine stärkere Gewichtung der Regionalgeschichte in der laufenden fachdidaktischen Ausbildung an der CAU, wie sie sich seit ein paar Jahren hoffnungsvoll abzeichnet, können längerfristig die Weichen hin zu einer stärkeren Implementierung der Regionalgeschichte in den Geschichtsunterricht gestellt werden. Dies wäre sicher auch von Vorteil für die Einbeziehung des Mittelalters am regionalhistorischen Exempel. Lohnen würde sich dafür die mittelalterliche Geschichte Schleswig-Holsteins allemal!

\section{Autor}

\section{Oliver Auge}

Professor für Regionalgeschichte mit dem Schwerpunkt Schleswig-Holsteins in Mittelalter und Früher Neuzeit an der Christian-Albrechts-Universität zu Kiel. Seine Forschungsschwerpunkte sind die Regional- und Landesgeschichte Schleswig-Holsteins, Württembergs, Mecklenburgs und Pommerns, Kirchen-', Stadt- und Universitätsgeschichte, Sozial- und Verfassungsgeschichte des Mittelalters und der Frühen Neuzeit.

oauge@email.uni-kiel.de

70 Schulte, Landes- oder Regionalgeschichte (wie Anm. 31), 107.

71 Ludwig Steindorff (Hrsg.), 100 Jahre SHUG. Schleswig-Holsteinische Universitätsgesellschaft - Brücke zwischen Universität und Land. Husum 2019, 72-74. 